\title{
COMPARACIÓN ENTRE CLORHEXIDINA E HIPOCLORITO DE SODIO COMO SOLUCIONES DESINFECTANTES EN LA PRÁCTICA ENDODÓNTICA
}

\author{
COMPARATIVE STUDY BETWEEN CHLORHEXIDINE AND SODIUM HYPOCHLORITE \\ AS DISINFECTANT SOLUTIONS IN ENDODONTIC PRACTICE, REVIEW
}

\author{
Cesar Andrade ${ }^{1 a}$, David Bustamante ${ }^{1 a}$, Osmani Guevara ${ }^{1 b}$, Ana Armas ${ }^{1 c}$
}

\begin{abstract}
RESUMEN
El éxito de los tratamientos endodónticos depende tanto de una buena conformación de los conductos radiculares como de la correcta desinfección del espacio pulpar ya que los organismos que habitan en las paredes de los conductos radiculares pueden sobrevivir como monocultivos por lo que es indispensable además de la eliminación mecánica de los lipopolisacáridos es uso de irrigantes como el hipoclorito de sodio que es uno de los irrigantes más utilizados para la desinfección de los conductos radiculares ya que nos permite disolver tejidos orgánicos además de su fuerte actividad antimicrobiana, la clorhexidina que tiene características biocompatibles además de dos características útiles al momento de la desinfección de los conductos radiculares como los son la sustentabilidad y su acción antimicrobiana o el ácido etilendiaminotetraacetico (EDTA) que presenta características quelantes que nos permite la eliminación de la capa de frotis o barrillo dentinario producto de la instrumentación mecánica de los conductos radiculares además de favorecer la acción de otros irrigantes a llegar a los túbulos dentinarios y sus ramificaciones.
\end{abstract}

Palabras clave: Clorhexidina; hipoclorito de sodio; endodoncia. (Fuente:DeCS)

\section{ABSTRACT}

The success of endodontic treatments depends both on a good conformation of the root canals and on the correct disinfection of the pulp space, since the organisms that live in the walls of the root canals can survive as monocultures. Mechanics of lipopolysaccharides is the use of irrigators such as sodium hypochlorite which is one of the most widely used irrigators for the disinfection of root canals as it allows us to dissolve organic tissues in addition to their strong antimicrobial activity, chlorhexidine that has biocompatible characteristics in addition to Two characteristics useful at the time of disinfection of the root canal, such as sustainability and its antimicrobial action or ethylenediaminetetraacetic acid (EDTA), which has chelating characteristics that allow the elimination of the smear layer or dentin barrel product of mechanical instrumentation Of the root canals as well as favoring the action of other irrigators to reach the dentinal tubules and their ramifications.

Key words: Chlorhexidine; sodium hypochlorite; endodontics. (Source: MeSH NLM)

${ }^{c}$ Docente Universitario

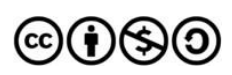

Correspondencia:

Osmani Fabricio Guevara Cabrera

Dirección: Quito-Ecuador-El Bosque, Marcos Joffre y Gerónimo Uretra, Oe12-43.

Teléfono: (+593) 0967024240

Correo electrónico: fabriche20@hotmail.com

Citar como: Andrade C, Bustamante D, Guevara O, Armas A. Comparación entre clorhexidina e hipoclorito de sodio como soluciones desinfectantes en la práctica endodóntica. KIRU. 2017;14(1):86-90. https://doi.org/10.24265/kiru.2017.v14n1.12 


\section{INTRODUCCIÓN}

Las bacterias y sus subproductos constituyen los agentes etiológicos responsables con más frecuencia de las infecciones endodónticas y lesiones periapicales $^{(1)}$, de ahí que su eliminación se muestra como uno de los principales objetivos de la preparación de los conductos radiculares ${ }^{(2)}$, considerando su compleja anatomía, la irrigación adecuada de estos, y una efectiva instrumentación, como elementos fundamentales en la reducción y/o eliminación de microorganismos de las zonas más inaccesibles de los conductos ${ }^{(1)}$.

En ese contexto, la presencia del Enterococcus faecalis ha sido reportada hasta en un $77 \%$ de los casos en dientes con lesiones endodónticas persistentes $^{(2)}$, evidenciando su papel fundamental en la etiología de infecciones periradiculares de conductos radiculares tratados ${ }^{3}$. Observamos que la eliminación completa de microorganismos de los conductos radiculares constituye una tarea compleja debido a su capacidad para penetrar en los túbulos dentinarios y su adaptabilidad a ambientes agresivos como la deficiencia de nutrientes, altas concentraciones de sal y ambiente alcalino extremo ${ }^{(1)}$.

En los últimos años es evidente el desarrollo de una variedad de soluciones de irrigación para el tratamiento de conductos ${ }^{1}$. El hipoclorito de sodio introducido como parte de los tratamientos endodónticos desde los años 30 se ha convertido en el irrigante de elección en estos procedimientos debido a su acción antimicrobiana potente y su capacidad para disolver el tejido necrótico pulpar(3). Su alto $\mathrm{pH}$ interfiere en la integridad de la membrana citoplasmática de la bacteria, desencadenando alteraciones en su metabolismo celular y destrucción de fosfolípidos ${ }^{(4)}$.

El gluconato de clorhexidina surge como una opción alterna en la irrigación de conductos debido a su potente acción antimicrobiana ${ }^{(3)}$ y elevada sustantividad al compararlo con el hipoclorito de sodio, su ausencia de olor y nula citotoxicidad; sin embargo, es manifiesta su incapacidad para eliminar los tejidos necróticos pulpares y la capa de barro dentinario de los conductos radiculares ${ }^{(2)}$. La ausencia de un consenso respecto a la sustancia ideal, asociada a la variedad de propiedades y características positivas o negativas de cada una de ellas, no permite definir la existencia de un único irrigante adecuado para tratar conductos radiculares durante tratamientos endodónticos ${ }^{(4)}$.

Así, conociendo que el objetivo principal del tratamiento endodóntico es lograr la eliminación de microorganismos residuales ${ }^{(2)}$, el presente trabajo pretende determinar, a través de una revisión bibliográfica, la efectividad del gluconato de clorhexidina y del hipoclorito de sodio como soluciones irrigantes empleadas en procedimientos endodónticos. Se plantea una revisión de literatura considerando artículos recuperados de bases de datos electrónicas de revistas biomédicas, PubMed, SCIELO, LILACS, Google académico, y se limita la búsqueda a artículos publicados desde el año 2008 al 2016. Utilizamos como palabras clave de búsqueda: clorhexidina, hipoclorito de sodio, endodoncia e irrigantes intraconductos. Fueron seleccionados estudios en seres humanos del tipo ensayos clínicos y estudios experimentales considerando diferentes microorganismos evaluados en combinación con hipoclorito de sodio y clorhexidina, así como estudios que consideraron la sustantividad de las sustancias desinfectantes. Del total de artículos obtenidos, 20 fueron seleccionados por cumplir con los criterios de inclusión determinados.

\section{Características de las soluciones desinfectantes irrigadoras de conductos}

En la desinfección de conductos radiculares, el hipoclorito de sodio evidencia una destacable reducción de la microbiota endodóntica en comparación con la clorhexidina ${ }^{(4)}$, efecto que se manifiesta cuando el hipoclorito de sodio es empleado en porcentajes de 5,25\% y en relación directa con las propiedades químicas ${ }^{(5)}$. Pese a esto, resulta aún muy discutible su efecto sobre los tejidos circundantes a la zona radicular(1). Por el contrario, evaluaciones realizadas comparando gluconato de clorhexidina en combinación con solución salina como opción de irrigante en dientes necróticos, se evidenció la capacidad de disolver la materia orgánica(6) por parte de esta sustancia.

Sin embargo, al comparar los efectos en cuanto a la capacidad de reducción de E. Faecalis de los conductos radiculares, entre hipoclorito de sodio al $2,5 \%$ y clorhexidina al $2 \%$ con aplicaciones de terapia fotodinámica en ambos, se evidenció un mayor efecto desinfectante del hipoclorito de sodio(2). Por otra parte, evaluando la sustantividad antimicrobiana de los diferentes irrigantes, gluconato de clorhexidina al $2 \%$, povidona yodada al $1 \%$, peróxido de hidrógeno al $2,5 \%$,seguido de hipoclorito de sodio al $2 \%$, hipoclorito de sodio al $2 \%$ y solución salina, se evidenció el mayor resultado en actividad antimicrobiana y sustantividad de la clorhexidina al $2 \%$ como irrigante durante 72 horas, recomendándolo para ser este utilizado como enjuague final en protocolos de tratamiento endodóntico $^{(7)}$. 
A nivel microbiológico no existen reportes de diferencias significativas entre hipoclorito de sodio al $2,5 \%$ y clorhexidina al $0,12 \%$, recomendando para la elección del irrigante en la terapia endodóntica considerar la indicación y propiedades de los mismos, más aun cuando la clorhexidina presenta la sustantividad que proporciona una desinfección que se prolonga de días a semanas, y que el hipoclorito de sodio posee gran capacidad de disolución de tejidos blandos y es efectivo cuando se trata de limpiar el sistema de conductos radiculares ${ }^{8}$. En contraste, otros estudios al evaluar la efectividad de diferentes sustancias sobre Cándida Albicans, microorganismos con más frecuencia encontrados en infecciones de conductos radiculares, el hipoclorito de sodio al 3\% mostró el mejor desempeño que el gluconato de clorhexidina al $2 \%$. Un resultado parecido obtuvo el EDTA (ácido etilendiaminotetraacético) que mostró una reducción menor del microorganismo evaluado ${ }^{(9)}$.

Comparando los efectos antimicrobianos del hipoclorito de sodio al 5,25\% y el gluconato de clorhexidina al $2 \%$, en combinación con activadores de irrigación sónicos y ultrasónicos (EndoActivator ${ }^{\circledR}$ o IRRI S(B) en 120 pacientes con periodontitis apical, se evidenció disminución de colonias de Enterococcus Faecalis mediante las técnicas combinadas de hipoclorito de sodio, activación sónica y ultrasónica en comparación con las técnicas combinadas de irrigación con clorhexidina y activación sónica y ultrasónica(3).

Similares resultados fueron reportados con el uso de EDTA al 17\%, clorhexidina al $2 \%$, empleándose el QMix como irrigante final-que al ser una solución irrigadora con agregado de detergente, con agentes tensoactivos, desencadena reducción de la tensión superficial, mejora la permeabilidad de la dentina radicular y permite una mejor penetración del mismo dentro del conducto-, sin diferencias significativas en cuanto a efectividad antibacteriana entre hipoclorito de sodio y clorhexidina(10). En estudios similares, al emplearse diferentes irrigantes, en forma combinada y consecutiva, fue evidente que estas combinaciones aumentaron sinérgicamente la acción asociada a la reacción ácido-base desencadenante; sin embargo, en ciertas ocasiones estas combinaciones conducen a la formación de precipitados con propiedades desfavorables $^{(9)}$.

Evaluando la eficacia antimicrobiana del extracto de hoja de guayaba y aloe vera en comparación con hipoclorito de sodio, clorhexidina y QMix empleados como irrigantes de conductos radiculares, observamos que si bien el extracto de hojas de guayaba y el extracto áloe vera poseen acciones antibacterianas y antioxidantes contra patógenos orales Candida Albicans y Enterococus Faecalis, los resultados muestran al QMix como una nueva propuesta para la irrigación de conductos relacionando su eficacia antibacteriana con su composición ${ }^{(11)}$.

En el mismo sentido, comparando la efectividad de agua ozonizada, hipoclorito de sodio y gluconato de clorhexidina en molares primarios infectados con Enterococus Faecalis bajo espectrómetro, se evidenció después de 6 horas un mejor desempeño de la clorhexidina como irrigante, en comparación con el hipoclorito de sodio, resultados que se mantuvieron durante las restantes 24 horas con una disminución posterior, recomendando la clorhexidina como irrigante de elección para el tratamiento de conductos en odontología pediátrica por su sustantividad y baja toxicidad. El agua ozonizada, por otro lado, también se evaluó y mostró ser una buena opción para el tratamiento de conductos en odontopediatría, requiriendo ser evaluada en su concentración y profundidad de acción ${ }^{(12)}$.

Evaluando la eficacia antimicrobiana de hipoclorito de sodio al 5,25\%, clorhexidina al $2 \%$, MTAD

-considerada como una mezcla de $3 \%$ de doxiciclina (un antibiótico de amplio espectro), 4,25\% de ácido cítrico) y un detergente (tween 80)-y de aplicaciones de láser de diodo a $830 \mathrm{~nm}$ en la eliminación de Enterococus Faecalis presente en conductos radiculares, se evidenció mejores resultados, en cuanto a desinfección, del hipoclorito de sodio al $5,25 \%$, seguido del efecto antimicrobiano de clorhexidina, sin diferencia significativa entre aplicaciones del láser de diodo y MTAD ${ }^{13}$. Resultados similares fueron reportados al evaluar la acción bactericida de la terapia fotodinámica (PDT), $2 \%$ de clorhexidina, $2,5 \% \mathrm{NaOCl}$ y la combinación de terapia fotoactivada proveniente de láser y $2,5 \% \mathrm{NaOCl}$ contra el mismo microorganismo, destacando una reducción al $100 \%$ de las colonias de Enterococus Faecalis con la combinación de Hipoclorito de sodio y $\operatorname{PDT}^{(14)}$.

La eficacia antibacteriana, antifúngica y antiviral del aceite esencial endémico Satureja Khuzistanica Jamzad, una planta endémica del sur de Irán utilizada por los nómadas para aliviar el dolor de dientes, fue evaluada frente a hipoclorito de sodio al $2,5 \%$ y gluconato de clorhexidina al $2 \%$, observándose que el aceite esencial evaluado puede ser tan eficaz en cuanto a desinfección como hipoclorito de sodio o clorhexidina contra patógenos resistentes, tales como Enterococus Faecalis, destacándose que a pesar de que los aceites esenciales se consideran generalmente como materiales seguros y en varios estudios evidencian biocompatibilidad con los tejidos vitales del huésped, es preciso considerar la complejidad del sistema radicular y la importancia de eliminar los restos de pulpa necrótica en las áreas en que la instrumentación mecánica tiene limitaciones, 
aconsejando hipoclorito de sodio sobre este nuevo irrigante $^{(15)}$. Un estudio similar, considerando $3 \%$ de hipoclorito de sodio, $2 \%$ de clorhexidina, $17 \%$ de EDTA y ácido etidrónico (18\%) frente a Candida Albicans, evidenció el efecto antimicrobiano medible incluso en presencia de la dentina que puede ser prometedor en la reducción del microorganismo en la terapia de conducto radicular del $\mathrm{NaOCl}$ y $\operatorname{EDTA}^{(16)}$.

Comparando los efectos antibacterianos de la irrigación con hipoclorito de sodio al 2,5\% o clorhexidina al $2 \%$, durante la preparación de los conductos radiculares infectados y preparados con o sin instrumentos de níquel-titanio rotatorios, se evidenció que ambos protocolos de irrigación fueron muy eficaces en la reducción de los niveles de especies de Streptococcus $(P<0,001)$, sin diferencia significativa con el uso o no de los instrumentos de rotación ${ }^{(17)}$.

Al comparar la eficacia de láser de baja potencia activado por la luz, $2 \%$ de clorhexidina y $2,5 \% \mathrm{NaOCl}$ en la eliminación de Enterococcus faecalis del sistema de conducto radicular, pudo concluirse que la terapia fotodinámica fue eficaz en la reducción de los recuentos de microorganismos evaluados en comparación con el grupo de control, pero la solución de $2,5 \% \mathrm{NaOCl}$ fue el protocolo más eficaz ${ }^{(18)}$.

Un estudio similar que evaluó la actividad antimicrobiana de hipoclorito de sodio al 5,25\%, clorhexidina al $2 \%$ Biopure MTAD, utilizado como procedimiento final en la eliminación del Enterococcus Faecalis, evidenció ausencia de diferencia significante entre las actividades antibacterianas de las sustancias evaluadas a los 5 minutos; sin embargo, a los dos días de irrigación, la actividad antibacteriana de Biopure MTAD se mostró tan eficaz como $\mathrm{NaOCl}$ y más eficaz que

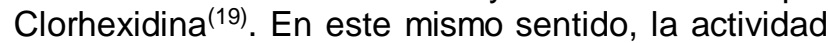
antibacteriana de las diferentes soluciones antibacterianas empleadas como irrigantes radiculares en los dientes con necrosis pulpar y con patología periapical fueron comparadas. Los resultados mostraron que tanto el gluconato de clorhexidina como el hipoclorito de sodio fueron eficaces en la reducción de los microorganismos en los dientes con pulpa necrótica, con patologías periapicales, o ambos ${ }^{(20)}$.

Evaluando la efectividad sobre la resistencia adhesiva tras la cementación de pernos en el canal radicular, empleando como protocolo de irrigación hipoclorito de sodio al $2,5 \%$ y clorhexidina al $2 \%$, intercaladas por alcohol isopropílico, solución salina o agua destilada como elementos empleados para prevenir la formación del precipitado, observamos que estas sustancias fracasaron en su misión de formar precipitado intracanal tras el uso de los dos irrigantes, pero que, sin embargo, las fuerzas de resistencia adhesiva probadas no se vieron afectadas $^{(21)}$.

Un trabajo similar evaluó la penetración del precipitado de cloranilina, formado tras el empleo de hipoclorito de sodio al 2,5\% como irrigante, seguido de EDTA, gluconato de clorhexidina, solución salina y QMiX -considerado como un nuevo irrigante compuesto por EDTA, gluconato de clorhexidina y un detergente no específico-, mediante espectrofotómetro de masa evidenciando la formación de este precipitado en la superficie y en el interior de los túbulos dentinarios irrigados con hipoclorito tras clorhexidina; sin embargo, este precipitado no fue detectado en los túbulos luego de la irrigación de hipoclorito seguido de solución salina o de $\mathrm{QMiX}^{(22)}$.

Un resultado similar fue reportado empleando cromatografía liquida, resonancia magnética con protones nucleares, espectroscopia, cromatografía a gas, cromatografía en lámina, prueba infrarroja, espectro microscopio, cromatografía a gas y espectrómetro de masa(23).

Se concluye con la literatura revisada evidencia que tanto el hipoclorito de sodio al 5,25\% como el gluconato de clorhexidina se muestran como soluciones de irrigación adecuadas en la desinfección en tratamientos endondónticos, con éxito a largo plazo. La literatura revisada muestra que el hipoclorito de sodio, al 5,25\% en solución, sigue siendo la mejor opción a utilizar para estas funciones, ya que gracias a sus propiedades consigue disolver la materia orgánica dentro de los conductos radiculares favoreciendo la limpieza de los mismos.

\section{Agradecimientos}

A las autoridades de la Universidad Tecnológica Equinoccial por el apoyo incondicional.

Contribuciones de autoría: $C A, D B, O C$ y $A A$ participaron en la revisión de la literatura, aporte de material de estudio y recolección de datos. La versión final del artículo fue aprobada por todos los participantes.

Fuente de financiamiento: Autofinanciado.

Conflictos de interés: Los autores declaran no tener conflictos de interés en esta publicación.

\section{REFERENCIAS BIBLIOGRÁFICAS}

1. Liu Y, Guo L, Li Y, Guo X, Wang B, Wu L. In vitro comparison of antimicrobial effectiveness of QMix and other final irrigants in human root canals. Sci Rep. 2015; 5: 17823. 
2. Samiei $M$, Shahi S,Ardalan-Abdollahi A Eskandarinezhad M, Negahdari R, Pakseresht Z. The Antibacterial Efficacy of Photo-Activated Disinfection, Chlorhexidine and Sodium Hypochlorite in Infected Root Canals: An in vitro study. Iran Endod J. 2016; 11(3):179-183.

3. Rico-Romano C,Zubizarreta-Macho A, BaqueroArtigao M, Mena-Álvarez J. An analysis in vivo of intracanal bacterial load before and after chemomechanical preparation: A comparative analysis of two irrigants and two activation techniques. J ClinExp Dent. 2016;8(1):e9-e13.

4. Karale R, Odebra KM, Srirekhaet A, Champa C, Shetty A, Pushpalathaet al.Effect of dentin on the antimicrobial efficacy of $3 \%$ sodium hypochlorite, $2 \%$ chlorhexidine, $17 \%$ ethylenediaminetetraacetic acid and $18 \%$ etidronic acid on Candida albicans: An in vitro study. J Conserv Dent. 2016;19:455-60. doi: 10.4103/0972-0707.190023.

5. Luddin N, Ahmed HMA. The antibacterial activity of sodium hypochlorite and chlorhexidine against Enterococcus faecalis: A review on agar diffusion and direct contact methods. J Conservative Dentistry. 2013; 16(1):9-16.

6. Da Silva EJNL, Monteiro MR, Belladonna FG, Almeida JF, De-Deus G, Neves AA. Postoperative Pain after Foraminal Instrumentation with a Reciprocating System and Different Irrigating Solutions. Braz Dent J. 2015; 26(3): 216-221.

7. Valera MC, Cardoso FGR, Chung A, Xavier ACC, Figueiredo MD, Martinho FC, Palo RM. Comparison of Different Irrigants in the Removal of Endotoxins and Cultivable Microorganisms from Infected Root Canals. The Scientific world Journal 2015. Article ID 125636: 16

8. Gonçalves LS, Rodrigues RCV, Andrade Junior CV, Soares RG, Vettore MV. The Effect of Sodium Hypochlorite and Chlorhexidine as Irrigant Solutions for Root Canal Disinfection: A Systematic Review of Clinical Trials. Journal of Endodontics 2016. 42 (4):527-532.

9. Shenoy A, Bolla N, Sayish, Sarath RK, Sunil-Ram CH, Sumlatha. Assessment of precipitate formation on interaction of irrigants used in different combinations: an in vitro study. Indian J Dent Res. 2013; 24:451-455. doi: 10.4103/0970-9290.118392.

10. Krishnamma S, Peedikayil F, Aman S, Tomy N, Pulickal-Mariodan J. Comparative Evaluation of Antimicrobial Activity of QMiX, 2.5\% Sodium Hypochlorite, 2\% Chlorhexidine, Guava Leaf Extract and Aloevera Extract Against Enterococcus faecalis and Candida albicans - An in-vitro Study. J ClinDiagn Res. 2016; 10 (5):ZC20-ZC23.

11. Goztas $Z$, Onat $H$, Tosun $G$, Sener $Y$, Huseyin $H$. Antimicrobial effect of ozonated water, sodium hypochlorite and chlorhexidinegluconate in primary molar root canals. Eur J Dent. 2014;8(4):469-474.

12. Ashofteh K, Sohrabi K, Iranparvar K, Chiniforush N. In vitro comparison of the antibacterial effect of three intracanalirrigants and diode laser on root canals infected with Enterococcus faecalis. Iran J Microbiol. $2014 ; 6(1): 26-30$
13. Elakanti S, Cherukuri G, Rao V, Chandrasekhar V, Rao A, Tummala M. Comparative evaluation of antimicrobial efficacy of $\mathrm{QMix}^{\mathrm{TM}} 2$ in 1 , sodium hypochlorite, and chlorhexidine against Enterococcus faecalis and Candida albicans. J Conserv Dent. 2015; 18(2):128-131.

14. Vaziri S, Kangarlou A, Shahbazi R, Nazari-Nasab A, Naseri M. Comparison of the bactericidal efficacy of photodynamic therapy, $2.5 \%$ sodium hypochlorite, and $2 \%$ chlorhexidine against Enterococcousfaecalis in root canals; an in vitro study. Dent Res J. 2012; 9(5):613-618.

15. Mollashahi NF, Bokaeian M, Mollashahir LF, Afrougheh A. Antifungal Efficacy of Green Tea Extract against Candida Albicans Biofilm on Tooth Substrate. $J$ Dentistry, Tehran University of Medical Sciences2015; 12(8):592-598.

16. Karale R, Odedra KM, Srirekha A, Champa C, Shetty A, Pushpalatha $S$, et al.Effect of dentin on the antimicrobial efficacy of $3 \%$ sodium hypochlorite, $2 \%$ chlorhexidine, $17 \%$ ethylenediaminetetraacetic acid, and $18 \%$ etidronic acid on Candida albicans: An in vitro study. J Conserv Dent. 2016; 19(5):455-60. doi: 10.4103/0972-0707.190023.

17. Rôças IN, Provenzano JC, Neves MA, Siqueira JF Jr. Disinfecting Effects of Rotary Instrumentation with Either $2.5 \%$ Sodium Hypochlorite or $2 \%$ Chlorhexidine as the Main Irrigant: A Randomized Clinical Study. J Endod.2016 Jun; 42(6):943-7. doi: 10.1016

18. Samiei M, Shahi S, Abdollahi AA, Eskandarinezhad M, Negahdari R, Pakseresht Z. La Eficacia antibacteriana de Activado-Foto Desinfección, clorhexidina y el hipoclorito de sodio en infectados Tratamientos de conducto: Estudio in vitro. Irán Endod J. 2016;11(3):179-83.

19. Agrawal V, Rao MR, Dhingra K, Gopal VR, Mohapatra A, Mohapatra A. Una comparación in vitro de effcacy antimicrobiana de tres irrigantes- Biopure conducto radicular MTAD, $2 \%$ de clorhexidina gluconato y $5,25 \%$ de hipoclorito de sodio como un enjuague final contra $E$. faecalis.J ContempDentPract. 2013; 14(5):842-7.

20. Ercan E, Ozekinci T, atakul F, Gül K.La actividad antibacteriana de $2 \%$ de clorhexidina gluconato y $5,25 \%$ de hipoclorito de sodio en canal de la raíz infectada: estudio in vivo. J Endod. 2004; 30(2):84-7.

21. Magro, MG, Kuga MC, Aranda- Garcia AJ, ChávezAndrade GM, Faria G, Keine KC, Só VR. Effectiveness of several solutions to prevent the formation of precipitate due to the interaction between sodium hypochlorite and chlorhexidine and its effect on bond strebht of an epoxy- based sealer. International Endodontics Journal, 2014 pg. 1- 6.

22. Kolosowski KP, Sodhi RNS, Anil Kisken, Basrani BR. Qualitative analysis of precipitate formation on the surface and in the tubules of dentin irrigated with sodium hypochlorite and a final rinse of chlorhexidine or QMIX. JOE. 2014. 40(12):2036-2040.

23. Orban EO, Irmak O, Hur D, Yaman BC, Karabucak B. Does para-chloroaniline really form after mixing sodium hypochlorite and chlorhexidine? JOE. 2016, 42(3): 455- 459 